\title{
EQUIVALENCE AND REDUCTION OF DELAY-DIFFERENTIAL SYSTEMS
}

\author{
MOHAMED SALAH BOUDELLIOUA \\ Department of Mathematics and Statistics \\ Sultan Qaboos University, PO Box 36 \\ Al-Khodh, 123, Muscat, Oman \\ e-mail: boudell@squ.edu.om
}

\begin{abstract}
A new direct method is presented which reduces a given high-order representation of a control system with delays to a firstorder form that is encountered in the study of neutral delay-differential systems. Using the polynomial system description (PMD) setting due to Rosenbrock, it is shown that the transformation connecting the original PMD with the first-order form is Fuhrmann's strict system equivalence. This type of system equivalence leaves the transfer function and other relevant structural properties of the original system invariant.
\end{abstract}

Keywords: polynomial matrix description, neutral delay-differential systems, strict system equivalence, determinantal ideals, Gröbner bases

\section{Introduction}

One of the most basic procedures in control systems theory is to transform a given system of differential or difference equations into a low-order form which is simpler but equivalent to the original system. The objective is to make identification, analysis and synthesis easier. The transformation involved, however, must preserve the relevant system properties such as controllability, observability and minimality if the conclusions about the reduced system are to remain valid about the original one. For systems described by ordinary differential or difference equations, Rosenbrock (1970) introduced the concept of polynomial matrix description (PMD). The resulting matrix has elements which are polynomials in a single indeterminate. He also introduced the notion of strict-system-equivalence between polynomial system matrices. However, a more general definition of strict-system-equivalence was given by Fuhrmann (1977) and this may be regarded mathematically as the equivalence of two polynomial system matrices through the multiplication of zero coprime matrices. For delay-differential control systems, the resulting PMD is a two-variable polynomial matrix, i.e. it has elements which are polynomials in two indeterminates. In fact, over the recent years, multivariate polynomial matrices have found many applications in $n$-D circuits, systems, controls, signal processing and other areas.
The problem of reducing a two-variable PMD to a first order form describing delay-differential systems was first studied by Pugh et al. (1998a). Their method involves the construction of certain block polynomial matrices from which factors have to be removed to ensure that the resulting transformations are polynomial. Furthermore, the equivalence transformation involved could not be set out directly in terms of the given initial polynomial matrix. A two-stage method for the reduction of a two-variable polynomial matrix to first order form used in the study of singular Roesser type 2-D discrete systems was given by Pugh et al. (2005a), and for singular Fornasini-Marchesini type 2-D discrete systems a direct method was given by Boudellioua (2006). In this paper, a new direct method similar to the one given in (Pugh et al., 2005a; Boudellioua, 2006) is proposed for a class of delay-differential systems. The resulting PMD is one which arises in the theory of neutral delay-differential systems as studied by Byrnes et al. (1984). The transformation connecting the original and final PMDs is shown to be that of Fuhrmann's strict system equivalence (F-SSE). This type of equivalence, also referred to as zero coprime system equivalence, was studied in the context of multidimensional systems by Levy (1981), Johnson (1993) and Pugh et al. (1996), and was shown to preserve many of the relevant original system properties. Furthermore, it was shown by Pugh et al. (1998b; 2005a) and Boudellioua 
(2006) that this transformation plays an important role in a number of areas of multidimensional systems theory.

\section{Polynomial Matrix Descriptions and Equivalence}

Consider the following first order system described by the following neutral delay-differential equations given by Byrnes et al. (1984):

$$
\begin{aligned}
\sum_{i=0}^{p} E_{i} \dot{x}(t-i h) & =\sum_{i=0}^{w} A_{i} x(t-i h)+\sum_{j=0}^{l} B_{j} u(t-j h), \\
y(t) & =\sum_{k=0}^{r} C_{k} x(t-k h)+\sum_{n=0}^{v} D_{n} u(t-n h),
\end{aligned}
$$

where $x(t)$ is the state vector, $u(t)$ denotes the input vector, $y(t)$ signifies the output vector, $h$ is a positive real constant, the matrices $E_{i}, A_{i}, B_{j}, C_{k}$ and $D_{n}$ are constant real matrices of appropriate dimensions and $E_{i}$ may be singular. Then the system (1) can be written in the polynomial matrix form

$$
\left[\begin{array}{cc}
\rho E(\sigma)-A(\sigma) & B(\sigma) \\
-C(\sigma) & D(\sigma)
\end{array}\right]\left[\begin{array}{r}
x(t) \\
-u(t)
\end{array}\right]=\left[\begin{array}{c}
0 \\
-y(t)
\end{array}\right],
$$

where $\rho=\mathrm{d} / \mathrm{d} t$ denotes the differential operator and $\sigma$ a backward shift operator, i.e., $\sigma x(t)=x(t-h)$, and $E(\sigma)$ may be singular.

The polynomial matrix

$$
P_{F}(\rho, \sigma)=\left[\begin{array}{c|c}
\rho E(\sigma)-A(\sigma) & B(\sigma) \\
\hline-C(\sigma) & D(\sigma)
\end{array}\right]
$$

is called a polynomial matrix description (PMD) in the first-order form associated with the system (1).

A more general description than (3) having an arbitrary but fixed order is given by the PMD

$$
P(\rho, \sigma)=\left[\begin{array}{rr}
T(\rho, \sigma) & U(\rho, \sigma) \\
-V(\rho, \sigma) & W(\rho, \sigma)
\end{array}\right],
$$

where $T(\rho, \sigma), U(\rho, \sigma), V(\rho, \sigma)$ and $W(\rho, \sigma)$ are $r \times p$, $r \times n, m \times p$ and $m \times n$ polynomial matrices, respectively.

A matrix description (4) in which $T(\rho, \sigma)$ has full row rank and $V(\rho, \sigma)=H(\rho, \sigma) T(\rho, \sigma)$ for some rational matrix $H(\rho, \sigma)$ is called an admissible matrix description and its transfer function is

$$
G(\rho, \sigma)=H(\rho, \sigma) U(\rho, \sigma)+W(\rho, \sigma) .
$$

A basic transformation proposed for the study of systems described by (4) is Fuhrmann's strict system equivalence (F-SSE) as given by Levy (1981) and Johnson
(1993). This transformation is based on zero coprime equivalence (ZC-E) characterized by the following definition.

Definition 1. Two polynomial matrices $P_{1}(\rho, \sigma)$ and $S_{1}(\rho, \sigma)$, of appropriate dimensions, are said to be zero left coprime (ZLC) if the matrix

$$
\left[P_{1}(\rho, \sigma) \quad S_{1}(\rho, \sigma)\right]
$$

has full rank for all $(\rho, \sigma) \in \mathbb{C}^{2}$. Similarly, $P_{2}(\rho, \sigma)$ and $S_{2}(\rho, \sigma)$ of appropriate dimensions are said to be zero right coprime $(\mathrm{ZRC})$ if the matrix

$$
\left[\begin{array}{ll}
P_{2}^{T}(\rho, \sigma) & S_{2}^{T}(\rho, \sigma)
\end{array}\right]^{T}
$$

has full rank for all $(\rho, \sigma) \in \mathbb{C}^{2}$.

As in the ordinary-differential case, the zero structure of a multidimensional system is a crucial indicator of system behavior. It was shown by Zerz (2000) that the controllability and observability of a multidimensional system is strongly connected to the zero structure of the associated polynomial matrix. This zero structure is captured by the determinantal ideals defined by the following.

Definition 2. The $i$-th order determinantal ideal $\mathcal{I}_{i}^{[P]}$ of a polynomial matrix $P(\rho, \sigma) \in \mathbb{R}^{r_{1} \times r_{2}}[\rho, \sigma]$ is defined to be the ideal generated by the $i$-th order minors of $P(\rho, \sigma)$.

The determinantal ideals

$$
\mathcal{I}_{i}^{[P]}, \quad i=1,2, \ldots, \min \left(r_{1}, r_{2}\right)
$$

satisfy the following inclusion:

$$
\mathbb{R}[\rho, \sigma] \supseteq \mathcal{I}_{1}^{[P]} \supseteq \mathcal{I}_{2}^{[P]} \supseteq \ldots \mathcal{I}_{\mu}^{[P]},
$$

where $\mu$ is the normal rank of $P(\rho, \sigma)$.

Example 1. Consider the two-variable polynomial matrix given by

$$
P(\rho, \sigma)=\left[\begin{array}{ccc}
\rho^{2} & 0 & \rho \\
0 & \sigma^{2} & \rho \sigma
\end{array}\right] .
$$

The ideals generated by the first and second order minors are

$$
\begin{aligned}
& \mathcal{I}_{2}^{[P]}=\left\langle\rho^{3} \sigma, \rho \sigma^{2}\right\rangle, \\
& \mathcal{I}_{1}^{[P]}=\left\langle\rho, \sigma^{2}\right\rangle .
\end{aligned}
$$

It is clear that $\mathcal{I}_{1}^{[P]} \supset \mathcal{I}_{2}^{[P]}$ as every element of $\mathcal{I}_{2}^{[P]}$ can be expressed in terms of elements of $\mathcal{I}_{1}^{[P]}$.

Definition 3. Let $\mathbb{P}(m, n)$ denote the class of $(r+m) \times$ $(r+n)$ admissible PMDs where $m, n$ are fixed positive integers and $r>-\min (m, n)$. Two PMDs $P_{1}(\rho, \sigma)$ and 
$P_{2}(\rho, \sigma)$ are said to be $Z C$ - $E$ if there exist polynomial matrices $S_{1}(\rho, \sigma), S_{2}(\rho, \sigma)$ of appropriate dimensions such that

$$
S_{2}(\rho, \sigma) P_{1}(\rho, \sigma)=P_{2}(\rho, \sigma) S_{1}(\rho, \sigma),
$$

where $P_{1}(\rho, \sigma), S_{1}(\rho, \sigma)$ are ZLC and $P_{2}(\rho, \sigma), S_{2}(\rho, \sigma)$ are ZRC.

In (Pugh et al., 1996; Pugh et al., 2005b), it was shown that ZC-E reveals some fundamental algebraic properties amongst its invariants.

Lemma 1. (Pugh et al., 2005b) Suppose that two polynomial matrices $P(\rho, \sigma)$ and $Q(\rho, \sigma) \in \mathbb{P}(m, n)$ are $Z C$-E. Let

$$
\mathcal{I}_{j}^{[P]}, \quad \forall j=1, \ldots, h=\min \left(r^{[P]}+m, r^{[P]}+n\right),
$$

denote the determinantal ideal of order $j$ generated by the $j \times j$ minors of $P(\rho, \sigma)$, and

$$
\mathcal{I}_{i}^{[Q]}, \quad \forall i=1, \ldots, k=\min \left(r^{[Q]}+m, r^{[Q]}+n\right),
$$

denote the determinantal ideal of order $i$ generated by the $i \times i$ minors of $Q(\rho, \sigma)$.

Then

$$
\mathcal{I}_{h-i}^{[P]}=\mathcal{I}_{k-i}^{[Q]}, \quad \forall i=0, \ldots, \bar{h},
$$

where $\bar{h}=\min (h-1, k-1)$, and

$$
\mathcal{I}_{h-i}^{[P]}=\langle 1\rangle, \quad \forall i>h
$$

or

$$
\mathcal{I}_{k-i}^{[Q]}=\langle 1\rangle \quad \text { if } \quad i<h \text { or } i<k .
$$

The transformation of F-SSE, which is a special case of $\mathrm{ZC}-\mathrm{E}$, is used in the reduction of multivariate PMDs and is defined by the following.

Definition 4. Two polynomial system matrices $P_{1}(\rho, \sigma)$ and $P_{2}(\rho, \sigma) \in \mathbb{P}(m, n)$ are said to be $F-S S E$ if they are related by the following:

$$
\begin{aligned}
& \underbrace{\left[\begin{array}{cc}
M(\rho, \sigma) & 0 \\
X(\rho, \sigma) & I_{m}
\end{array}\right]}_{S_{1}(\rho, \sigma)} \underbrace{\left[\begin{array}{rr}
T_{1}(\rho, \sigma) & U_{1}(\rho, \sigma) \\
-V_{1}(\rho, \sigma) & W_{1}(\rho, \sigma)
\end{array}\right]}_{P_{2}(\rho, \sigma)} \\
& =\underbrace{\left[\begin{array}{cc}
T_{2}(\rho, \sigma) & U_{2}(\rho, \sigma) \\
-V_{2}(\rho, \sigma) & W_{2}(\rho, \sigma)
\end{array}\right]}_{P_{1}(\rho, \sigma)} \underbrace{\left[\begin{array}{cc}
N(\rho, \sigma) & Y(\rho, \sigma) \\
0 & I_{n}
\end{array}\right]}_{S_{2}(\rho, \sigma)},
\end{aligned}
$$

where $P_{1}(\rho, \sigma), S_{1}(\rho, \sigma)$ are ZLC, $P_{2}(\rho, \sigma), S_{2}(\rho, \sigma)$ are $\mathrm{ZRC}$, and $M(\rho, \sigma), N(\rho, \sigma), X(\rho, \sigma)$ and $Y(\rho, \sigma)$ are polynomial matrices of appropriate dimensions.
In the case when the system matrices $P_{1}(\rho, \sigma), P_{2}(\rho, \sigma)$ have the same size and the matrices $M(\rho, \sigma), N(\rho, \sigma)$ are unimodular, the transformation in (17) is called Rosenbrock's strict system equivalence (R-SSE). Clearly, R-SSE is a special case of F-SSE. In fact, the latter can be generated from the former together with a trivial expansion or deflation of the PMDs. However, it should be pointed out here that, unlike in the single variable case, two PMDs which are R-SSE cannot be always obtained from each other by a finite sequence of elementary row or column operations, see (Sebek, 1988).

Lemma 2. (Johnson, 1993) The transformation of F-SSE given in (17) preserves the transfer function of $P(\rho, \sigma)$ and, in the sense described in Lemma 1, the determinantal ideals of the matrices

$$
T(\rho, \sigma), P(\rho, \sigma),[T(\rho, \sigma) \quad U(\rho, \sigma)],\left[\begin{array}{r}
T(\rho, \sigma) \\
-V(\rho, \sigma)
\end{array}\right] .
$$

\section{Reduction to the First Order}

Let $P(\rho, \sigma)$ be a two-variable PMD given by (4), with $s=r+m$ and $z=r+n$. First write $P(\rho, \sigma)$ as

$$
\begin{aligned}
P(\rho, \sigma) & =\sum_{i=0}^{q} P_{i}(\sigma) \rho^{i} \\
& =P_{0}(\sigma) \rho^{0}+P_{1}(\sigma) \rho^{1}+\cdots+P_{q}(\sigma) \rho^{q},
\end{aligned}
$$

where $P_{i}(\sigma), i=0,1, \ldots, q$ are $s \times z$ polynomial matrices and $q=\operatorname{deg}_{\rho} P(\rho, \sigma)$, i.e., the degree of $P(\rho, \sigma)$ in $\rho$.

Now construct the block polynomial matrices

$$
E(\sigma)=\left[\begin{array}{cccccc}
0 & -I_{z} & \ldots & 0 & 0 & 0 \\
0 & 0 & \ldots & 0 & 0 & 0 \\
\vdots & \vdots & \ddots & \vdots & \vdots & \vdots \\
0 & 0 & \ldots & 0 & -I_{z} & 0 \\
P_{q}(\sigma) & P_{q-1}(\sigma) & \ldots & P_{2}(\sigma) & P_{1}(\sigma) & 0 \\
0 & 0 & \ldots & 0 & 0 & 0
\end{array}\right]
$$

and

$$
A(\sigma)=\left[\begin{array}{cccccc}
-I_{z} & 0 & \ldots & 0 & 0 & 0 \\
0 & -I_{z} & \ldots & 0 & 0 & 0 \\
\vdots & \vdots & \ddots & \vdots & \vdots & \vdots \\
0 & 0 & \ldots & -I_{z} & 0 & 0 \\
0 & 0 & \ldots & 0 & -P_{0}(\sigma) & Z_{m} \\
0 & 0 & \ldots & 0 & Z_{n} & 0
\end{array}\right],
$$

where $Z_{n}=\left[\begin{array}{ll}0_{n, r} & I_{n}\end{array}\right]$ and $Z_{m}^{T}=\left[\begin{array}{cc}0_{m, r} & I_{m}\end{array}\right]$. 
Theorem 1. Let $P(\rho, \sigma)$ be a PMD given by (19) and the matrices $E(\sigma)$ and $A(\sigma)$ be constructed as in (20) and (21), respectively. Then the $[(z q+m)+m] \times$ $[(z q+m)+n] P M D$ in the first-order form (3)

$$
Q(\rho, \sigma)=\left[\begin{array}{c|c}
\rho E(\sigma)-A(\sigma) & {\left[\begin{array}{c}
0_{(z q+m-n), n} \\
I_{n}
\end{array}\right]} \\
\hline\left[\begin{array}{ll}
0_{m, z q} & -I_{m}
\end{array}\right] & 0
\end{array}\right],
$$

is F-SSE with $P(\rho, \sigma)$.

Proof. First prove that $Q(\rho, \sigma)$ is related to the original PMD, $P(\rho, \sigma)$, by the following:

$$
S_{1}(\rho, \sigma) P(\rho, \sigma)=Q(\rho, \sigma) S_{2}(\rho, \sigma),
$$

where

$$
S_{1}(\rho, \sigma)=\left[\begin{array}{c|c}
0_{z(q-1), r} & 0_{z(q-1), m} \\
I_{r} & 0_{r, m} \\
0_{(m+n), r} & 0_{(m+n), m} \\
\hline 0_{m, r} & I_{m}
\end{array}\right],
$$

and

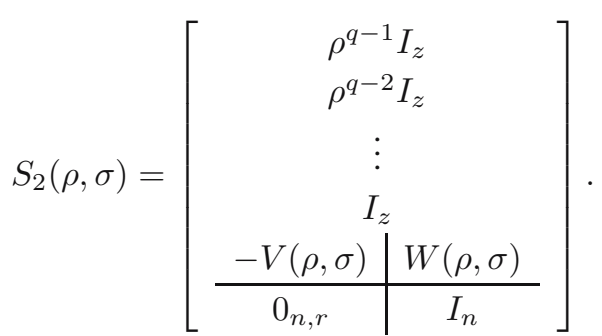

The matrix $Q(\rho, \sigma)$ in $(22)$ can be represented in the form

$$
Q(\rho, \sigma)=\left[\begin{array}{cccccc|c}
I_{z} & -\rho I_{z} & \cdots & 0 & 0 & 0 & 0 \\
0 & I_{z} & \cdots & 0 & 0 & 0 & 0 \\
\vdots & \vdots & \ddots & \vdots & \vdots & \vdots & \vdots \\
0 & 0 & \cdots & I_{z} & -\rho I_{z} & 0 & 0 \\
\alpha & \beta & \cdots & \gamma & \delta & -Z_{m} & 0 \\
0 & 0 & \cdots & 0 & -Z_{n} & 0 & I_{n} \\
\hline 0 & 0 & \cdots & 0 & 0 & -I_{m} & 0
\end{array}\right]
$$

where $\alpha=\rho P_{q}(\sigma), \beta=\rho P_{q-1}(\sigma), \gamma=\rho P_{2}(\sigma), \delta=$ $\rho P_{1}(\sigma)+P_{0}(\sigma)$.

From this it can be easily deduced that

$$
\begin{aligned}
S_{1}(\rho, \sigma) P(\rho, \sigma)= & Q(\rho, \sigma) S_{2}(\rho, \sigma) \\
& =\left[\begin{array}{cc}
0_{z(q-1), r} & 0_{z(q-1), n} \\
T(\rho, \sigma) & U(\rho, \sigma) \\
0_{m+n, r} & 0_{m+n, n} \\
-V(\rho, \sigma) & W(\rho, \sigma)
\end{array}\right] .
\end{aligned}
$$

Now it remains to prove that $Q(\rho, \sigma), S_{1}(\rho, \sigma)$ are ZLC and $P(\rho, \sigma), S_{2}(\rho, \sigma)$ are ZRC. This follows from the fact that the highest-order minor of the matrix

$$
\left[Q(\rho, \sigma) S_{1}(\rho, \sigma)\right],
$$

obtained by deleting the columns $z(q-1)+1, \ldots, z q$,

$$
\left|\begin{array}{ccccccc}
I_{z} & -\rho I_{z} & \cdots & 0 & 0 & 0 & 0 \\
0 & I_{z} & \cdots & 0 & 0 & 0 & 0 \\
\vdots & \vdots & \ddots & \vdots & \vdots & \vdots & \vdots \\
0 & 0 & \cdots & I_{z} & 0 & 0 & 0 \\
\rho P_{q}(\sigma) & \rho P_{q-1}(\sigma) & \cdots & \rho P_{2}(\sigma) & 0 & E_{r} & 0 \\
0 & 0 & \cdots & 0 & I_{n} & 0 & 0 \\
0 & 0 & \cdots & 0 & 0 & 0 & I_{m}
\end{array}\right|,
$$

where $E_{r}^{T}=\left[\begin{array}{cc}I_{r} & 0_{r, m}\end{array}\right]$, is equal to \pm 1 .

Similarly, the matrix

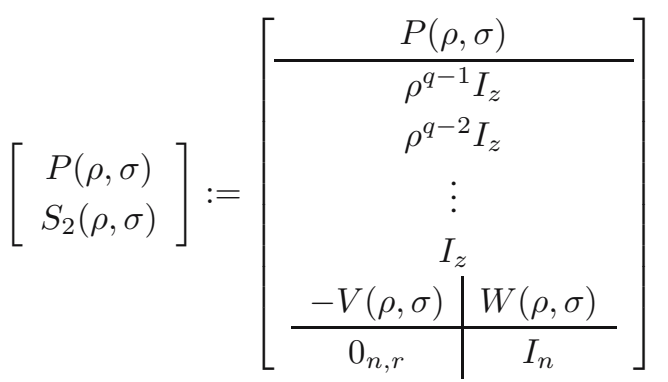

clearly contains a block identity matrix $I_{z}$, therefore it has a highest order minor equal to 1 .

It is interesting to note that the resulting first-order form obatined in (22) involves delays only in the state variables. Furthermore, from the computational aspect, $Q(\sigma, \rho)$ can be obtained from $P(\rho, \sigma)$ in a simple manner, as shown by the following result.

Theorem 2. The first-order form $Q(\sigma, \rho)$ in (22) can be obtained from $P(\rho, \sigma)$ in (4) by using a trivial expansion on $P(\rho, \sigma)$ followed by a finite sequence of elementary row and column operations.

Proof. Let $P_{E}(\rho, \sigma)$ denote the trivial expansion of $P(\rho, \sigma)$, such that $P_{E}(\rho, \sigma)$ have the same size as $Q(\rho, \sigma)$, i.e.,

$$
P_{E}(\rho, \sigma)=\left[\begin{array}{cc}
I_{z q+m-r} & 0 \\
0 & P(\rho, \sigma)
\end{array}\right] .
$$

Clearly, $P_{E}(\rho, \sigma)$ and $P(\rho, \sigma)$ are F-SSE since they are related by the following transformation:

$$
\left[\begin{array}{c}
0 \\
I_{r+n}
\end{array}\right] P(\rho, \sigma)=P_{E}(\rho, \sigma)\left[\begin{array}{c}
0 \\
I_{r+m}
\end{array}\right] .
$$


Now let $P_{N}(\rho, \sigma)$ denote the normalized PMD of $P(\rho, \sigma)$ given by

$$
\begin{aligned}
& P_{N}(\rho, \sigma)=\left[\begin{array}{ccc|c}
I & 0 & 0 & 0 \\
0 & P(\rho, \sigma) & -Z_{m} & 0 \\
0 & -Z_{n}^{T} & 0 & I_{n} \\
\hline 0 & 0 & I_{m} & 0
\end{array}\right] \\
& :=\left[\begin{array}{cccc|c}
I & 0 & 0 & 0 & 0 \\
0 & T(\rho, \sigma) & U(\rho, \sigma) & 0 & 0 \\
0 & -V(\rho, \sigma) & W(\rho, \sigma) & I_{m} & 0 \\
0 & 0 & -I_{n} & 0 & I_{n} \\
\hline 0 & 0 & 0 & I_{m} & 0
\end{array}\right] .
\end{aligned}
$$

$P_{E}(\rho, \sigma)$ in (30) and $P_{N}(\rho, \sigma)$ in (32) are related by the following R-SSE:

$$
P_{E}(\rho, \sigma)=E_{L}(\rho, \sigma) P_{N}(\rho, \sigma) E_{R}(\rho, \sigma)
$$

where

$$
E_{L}(\rho, \sigma)=\left[\begin{array}{cccc|c}
I & 0 & 0 & 0 & 0 \\
0 & 0 & -I & -W(\rho, \sigma) & 0 \\
0 & 0 & 0 & -I & 0 \\
0 & I & 0 & U(\rho, \sigma) & 0 \\
\hline 0 & 0 & I & W(\rho, \sigma) & I
\end{array}\right]
$$

and

$$
E_{R}(\rho, \sigma)=\left[\begin{array}{cccc|c}
I & 0 & 0 & 0 & 0 \\
0 & 0 & 0 & I & 0 \\
0 & 0 & I & 0 & I \\
0 & I & 0 & -V(\rho, \sigma) & W(\rho, \sigma) \\
\hline 0 & 0 & 0 & 0 & I
\end{array}\right]
$$

Clearly, $E_{L}(\rho, \sigma)$ and $E_{R}(\rho, \sigma)$ are unimodular and can be generated by a finite sequence of elementary row and column operations, respectively, since each contains a block identity matrix in all rows and in all column positions.

On the other hand, $P_{N}(\rho, \sigma)$ and $Q(\rho, \sigma)$ are related by the R-SSE

$$
P_{N}(\rho, \sigma)=F_{L}(\rho, \sigma) Q(\rho, \sigma) F_{R}(\rho, \sigma),
$$

where

$$
F_{L}(\rho, \sigma)=\left[\begin{array}{cccccc|c}
I & 0 & \ldots & 0 & 0 & 0 & 0 \\
0 & 0 & \ldots & 0 & 0 & 0 & 0 \\
\vdots & \vdots & \ddots & \vdots & \vdots & \vdots & \vdots \\
0 & 0 & \ldots & I & 0 & 0 & 0 \\
-\rho P_{q}(\sigma) & \zeta & \ldots & \xi & I & 0 & 0 \\
0 & 0 & \ldots & 0 & 0 & I & 0 \\
\hline 0 & 0 & \ldots & 0 & 0 & 0 & I
\end{array}\right],
$$

where $\zeta=-\rho P_{q-1}(\sigma), \xi=-\rho P_{q-2}(\sigma)$, and

$$
F_{R}(\rho, \sigma)=\left[\begin{array}{ccccc|c}
I & \rho & 0 & \ldots & 0 & 0 \\
0 & I & \rho & \ldots & 0 & 0 \\
\vdots & \vdots & \vdots & \ddots & \vdots & \vdots \\
0 & 0 & 0 & \ldots & \rho & 0 \\
0 & 0 & 0 & \ldots & I & 0 \\
\hline 0 & 0 & 0 & \ldots & 0 & I
\end{array}\right] .
$$

Again, $F_{L}(\rho, \sigma)$ and $F_{R}(\rho, \sigma)$ are unimodular matrices which can be generated by a finite sequence of elementary row and column operations, respectively, since each contains a block identity matrix in all rows and in all column positions.

It follows that $Q(\rho, \sigma)$ in (22) can be obtained from $P_{E}(\rho, \sigma)$ in (30) by a finite sequence of elementary row and column operations since they are related by the following R-SSE:

$$
\begin{aligned}
Q(\rho, \sigma)= & F_{L}^{-1}(\rho, \sigma) E_{L}^{-1}(\rho, \sigma) P_{E}(\rho, \sigma) \\
& \times E_{R}^{-1}(\rho, \sigma) F_{R}^{-1}(\rho, \sigma) .
\end{aligned}
$$

\section{Example}

Consider the single-input-single-output second-order system described by the following delay-differential equations:

$$
\begin{aligned}
\ddot{x}(t)= & -\ddot{x}(t-2 h)+2 \dot{x}(t-2 h)-\dot{x}(t-h) \\
& -3 \dot{x}(t)+\ddot{u}(t-2 h)-\ddot{u}(t-h) \\
& -\dot{u}(t-2 h)+2 \dot{u}(t)+u(t-2 h)-u(t-h), \\
y(t)= & -\ddot{x}(t-2 h)+2 \ddot{x}(t)+\dot{x}(t-2 h) \\
& -\dot{x}(t-h)+4 \dot{x}(t)+x(t)+2 \ddot{u}(t-2 h) \\
& -\ddot{u}(t-h)+5 \dot{u}(t-h)+u(t-2 h) \\
& -u(t-h)+3 u(t),
\end{aligned}
$$

where $x(t)$ is the state variable, $u(t)$ denotes the input variable, $y(t)$ signifies the output variable and $h$ is a positive real constant. Then the PMD associated with (40) is 
given by

$$
P(\rho, \sigma):=\left[\begin{array}{c|c}
T_{P}(\rho, \sigma) & U_{P}(\rho, \sigma) \\
\hline-V_{P}(\rho, \sigma) & W_{P}(\rho, \sigma)
\end{array}\right],
$$

where

$$
\begin{aligned}
T_{P}(\rho, \sigma)= & \left(\sigma^{2}+1\right) \rho^{2}-\left(2 \sigma^{2}-\sigma-3\right) \rho \\
& +\sigma^{2}-4 \sigma+1, \\
U_{P}(\rho, \sigma)= & \left(\sigma^{2}-\sigma\right) \rho^{2}-\left(\sigma^{2}-2\right) \rho+\sigma^{2}-\sigma, \\
V_{P}(\rho, \sigma)= & -(\sigma+2) \rho^{2}+\left(\sigma^{2}-\sigma\right) \rho+4 \sigma+1, \\
W_{P}(\rho, \sigma)= & \left(2 \sigma^{2}-\sigma\right) \rho^{2}+5 \sigma \rho+\sigma^{2}-\sigma+3 .
\end{aligned}
$$

Here $r=1, m=n=1, q=2$ and $s=z=2$.

Using Maple, the transfer function $G^{[P]}(\rho, \sigma)$ of $P(\rho, \sigma)$ is given by

$$
\begin{aligned}
G^{[P]} & (\rho, \sigma) \\
= & {\left[\left(\sigma^{2}+1\right) \rho^{2}-\left(2 \sigma^{2}-\sigma-3\right) \rho+\sigma^{2}-4 \sigma+1\right]^{-1} } \\
& \times\left[-\left(\sigma^{3}+\sigma^{2}-2 \sigma\right) \rho^{4}\right. \\
& +\left(\sigma^{4}-\sigma^{3}+3 \sigma^{2}-2 \sigma-4\right) \rho^{3}-\left(\sigma^{4}-4 \sigma^{3}+2 \sigma\right) \rho^{2} \\
& \left.+\left(\sigma^{4}-6 \sigma^{3}+13 \sigma+2\right) \rho+4 \sigma^{3}-2 \sigma^{2}-2 \sigma+3\right] .
\end{aligned}
$$

The reduced Gröbner bases of the determinantal ideals generated by the minors of the matrices in Lemma 1 associated with $P(\rho, \sigma)$ are given by

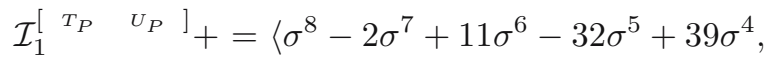

$$
\begin{aligned}
& -2 \sigma^{3}-19 \sigma^{2}-4 \sigma+4,16 \rho+3 \sigma^{7} \\
& -6 \sigma^{6}+35 \sigma^{5}-98 \sigma^{4}+137 \sigma^{3} \\
& \left.-50 \sigma^{2}-27 \sigma+10\right\rangle \text {, } \\
& \mathcal{I}_{1}^{\left[\begin{array}{r}
T_{P} \\
-V_{P}
\end{array}\right]}=\left\langle\sigma^{8}+17 \sigma^{6}-35 \sigma^{5}+22 \sigma^{4}+103 \sigma^{3}\right. \\
& -29 \sigma^{2}-114 \sigma-9 \text {, } \\
& 179880 \rho+1879 \sigma^{7}-1563 \sigma^{6} \\
& +37934 \sigma^{5}-93203 \sigma^{4}+202249 \sigma^{3} \\
& \left.-68516 \sigma^{2}-12719 \sigma+102237\right\rangle \text {, } \\
& \mathcal{I}_{1}^{[P]}=\langle 1\rangle, \\
& \mathcal{I}_{2}^{[P]}=\left\langle\left(2 \sigma^{4}-2 \sigma^{3}+\sigma^{2}+\sigma\right)\right. \\
& \times \rho^{4}\left(-3 \sigma^{4}+8 \sigma^{3}+8 \sigma^{2}-4\right) \rho^{3} \\
& +\left(2 \sigma^{4}-16 \sigma^{3}+13 \sigma^{2}+12 \sigma+3\right) \rho^{2} \\
& -\left(\sigma^{4}-2 \sigma^{3}+24 \sigma^{2}-13 \sigma-11\right) \rho \\
& \left.+\sigma^{4}-\sigma^{3}+5 \sigma^{2}-14 \sigma+3\right\rangle \text {. }
\end{aligned}
$$

Writing $P(\rho, \sigma)$ in the form (19), we have

$$
P(\rho, \sigma)=\underbrace{\left[\begin{array}{cc}
\sigma^{2}-4 \sigma+1 & \sigma^{2}-\sigma \\
-4 \sigma-1 & \sigma^{2}-\sigma+3
\end{array}\right]}_{P_{0}(\sigma)} \rho^{0}
$$

$$
\begin{aligned}
& +\underbrace{\left[\begin{array}{cc}
-2 \sigma^{2}+\sigma+3 & -\sigma^{2}+2 \\
-\sigma^{2}+\sigma & 5 \sigma
\end{array}\right]}_{P_{1}(\sigma)} \rho^{1} \\
& +\underbrace{\left[\begin{array}{cc}
\sigma^{2}+1 & \sigma^{2}-\sigma \\
\sigma+2 & 2 \sigma^{2}-\sigma
\end{array}\right]}_{P_{2}(\sigma)} \rho^{2} .
\end{aligned}
$$

Then, constructing the $6 \times 6 \mathrm{PMD}$ in the first-order form $Q(\rho, \sigma)$ corresponding to (22) gives

$$
\begin{aligned}
Q(\rho, \sigma) & :=\left[\begin{array}{cc}
T_{Q}(\rho, \sigma) & U_{Q}(\rho, \sigma) \\
-V_{Q}(\rho, \sigma) & W_{Q}(\rho, \sigma)
\end{array}\right] \\
& =\left[\begin{array}{ccc|c}
I_{2} & -\rho I_{2} & 0 & 0 \\
\rho P_{2}(\sigma) & \rho P_{1}(\sigma)+P_{0}(\sigma) & -Z_{1} & 0 \\
0 & -Z_{1}^{T} & 0 & 1 \\
\hline 0 & 0 & 1 & 0
\end{array}\right] .
\end{aligned}
$$

The matrices $E(\sigma)$ and $A(\sigma)$ corresponding respectively to (20) and (21) are given by

$$
\begin{aligned}
& E(\sigma)=\left[\begin{array}{ccc}
0 & -I_{2} & 0 \\
P_{2}(\sigma) & P_{1}(\sigma) & 0 \\
0 & 0 & 0
\end{array}\right], \\
& A(\sigma)=\left[\begin{array}{ccc}
-I_{2} & 0 & 0 \\
0 & -P_{0}(\sigma) & Z_{1} \\
0 & Z_{1}^{T} & 0
\end{array}\right] .
\end{aligned}
$$

By virtue of Theorem 1, the polynomial matrix $P(\rho, \sigma)$ in $(41)$ and the corresponding PMD in the firstorder form $Q(\rho, \sigma)$ in (44) are related by an F-SSE of the form (23), with

$$
\begin{aligned}
& S_{1}(\rho, \sigma)=\left[\begin{array}{c|c}
0_{2,1} & 0_{2,1} \\
1 & 0 \\
0_{2,1} & 0_{2,1} \\
\hline 0 & 1
\end{array}\right], \\
& S_{2}(\rho, \sigma)=\left[\begin{array}{c|c}
\multicolumn{2}{c}{\rho I_{2}} \\
I_{2} \\
-V_{P}(\rho, \sigma) & W_{P}(\rho, \sigma) \\
\hline 0 & 1
\end{array}\right] .
\end{aligned}
$$


In fact, it can be easily verified that

$$
\begin{aligned}
S_{1}(\rho, \sigma) P(\rho, \sigma) & =Q(\rho, \sigma) S_{2}(\rho, \sigma) \\
& =\left[\begin{array}{cc}
0_{2,1} & 0_{2,1} \\
T_{P}(\rho, \sigma) & U_{P}(\rho, \sigma) \\
0_{2,1} & 0_{2,1} \\
-V_{P}(\rho, \sigma) & W_{P}(\rho, \sigma)
\end{array}\right],
\end{aligned}
$$

where $T_{P}(\rho, \sigma), U_{P}(\rho, \sigma), V_{P}(\rho, \sigma)$ and $W_{P}(\rho, \sigma)$ are given by $(42)$.

The matrices $Q(\rho, \sigma), S_{1}(\rho, \sigma)$ are ZLC and the matrices $P(\rho, \sigma), S_{2}(\rho, \sigma)$ are ZRC since the matrix $\left[Q(\rho, \sigma) S_{1}(\rho, \sigma)\right]$ has the highest order minor

$$
\left|\begin{array}{ccccc}
I_{2} & 0_{2,1} & 0_{2,1} & 0_{2,1} & 0_{2,1} \\
\rho P_{2}(\sigma) & -Z_{1} & 0 & E_{2} & 0_{2,1} \\
0 & 0 & 1 & 0 & 0 \\
0 & 1 & 0 & 0 & 1
\end{array}\right|
$$

which is equal to 1 .

Similarly, the matrix

$$
\left[\begin{array}{c}
P(\rho, \sigma) \\
S_{2}(\rho, \sigma)
\end{array}\right]:=\left[\begin{array}{cc}
T_{P}(\rho, \sigma) & U_{P}(\rho, \sigma) \\
-V_{P}(\rho, \sigma) & W_{P}(\rho, \sigma) \\
\hline \rho & 0 \\
0 & \rho \\
1 & 0 \\
0 & 1 \\
-V_{P}(\rho, \sigma) & W_{P}(\rho, \sigma) \\
0 & 1
\end{array}\right]
$$

contains a block identity matrix and therefore has a highest order minor which is equal to 1 . by

The transfer function $G^{[Q]}(\rho, \sigma)$ of $Q(\rho, \sigma)$ is given

$$
\begin{aligned}
G^{[Q]} & (\rho, \sigma) \\
= & {\left[\left(\sigma^{2}+1\right) \rho^{2}-\left(2 \sigma^{2}-\sigma-3\right) \rho+\sigma^{2}-4 \sigma+1\right]^{-1} } \\
& \quad \times\left[-\left(\sigma^{3}+\sigma^{2}-2 \sigma\right) \rho^{4}\right. \\
& +\left(\sigma^{4}-\sigma^{3}+3 \sigma^{2}-2 \sigma-4\right) \rho^{3}-\left(\sigma^{4}-4 \sigma^{3}+2 \sigma\right) \rho^{2} \\
& \left.+\left(\sigma^{4}-6 \sigma^{3}+13 \sigma+2\right) \rho+4 \sigma^{3}-2 \sigma^{2}-2 \sigma+3\right] \\
= & G^{[P]}(\rho, \sigma)
\end{aligned}
$$

and the reduced Gröbner bases of the determinantal ideals generated by the minors of the matrices in Lemma 1 asso- ciated with $Q(\rho, \sigma)$ are given by

$$
\begin{aligned}
& \left.\mathcal{I}_{i}^{\left[T_{Q}\right.}{ }^{T_{Q}}\right]=\langle 1\rangle, \quad i=1, \ldots, 4, \\
& \left.\mathcal{I}_{5}^{\left[{ }^{T_{Q}}\right.}{ }^{U_{Q}}\right]=\left\langle\sigma^{8}-2 \sigma^{7}+11 \sigma^{6}-32 \sigma^{5}+39 \sigma^{4}\right. \text {, } \\
& -2 \sigma^{3}-19 \sigma^{2}-4 \sigma+4 \text {, } \\
& 16 \rho+3 \sigma^{7}-6 \sigma^{6}+35 \sigma^{5}-98 \sigma^{4} \\
& \left.+137 \sigma^{3}-50 \sigma^{2}-27 \sigma+10\right\rangle \\
& =\mathcal{I}_{1}^{\left[\begin{array}{ll}
T_{P} & U_{P}
\end{array}\right]}
\end{aligned}
$$

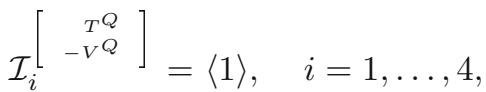

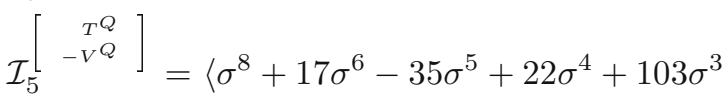

$$
\begin{aligned}
& -29 \sigma^{2}-114 \sigma-9 \text {, } \\
& 179880 \rho+1879 \sigma^{7}-1563 \sigma^{6} \\
& +37934 \sigma^{5}-93203 \sigma^{4}+202249 \sigma^{3} \\
& \left.-68516 \sigma^{2}-12719 \sigma+102237\right\rangle \\
& \left.=\mathcal{I}_{1}^{T_{P}^{T}}-V_{P}^{T}\right]^{T}, \\
& \mathcal{I}_{j}^{[Q]}=\langle 1\rangle, \quad j=1, \ldots, 4, \\
& \mathcal{I}_{5}^{[Q]}=\langle 1\rangle \\
& =\mathcal{I}_{1}^{[P]}, \\
& \mathcal{I}_{6}^{[Q]}=\left\langle\left(2 \sigma^{4}-2 \sigma^{3}+\sigma^{2}+\sigma\right) \rho^{4}\right. \\
& \times\left(-3 \sigma^{4}+8 \sigma^{3}+8 \sigma^{2}-4\right) \rho^{3} \\
& +\left(2 \sigma^{4}-16 \sigma^{3}+13 \sigma^{2}+12 \sigma+3\right) \rho^{2} \\
& -\left(\sigma^{4}-2 \sigma^{3}+24 \sigma^{2}-13 \sigma-11\right) \rho \\
& \left.+\sigma^{4}-\sigma^{3}+5 \sigma^{2}-14 \sigma+3\right\rangle \\
& =\mathcal{I}_{2}^{[P]} \text {, }
\end{aligned}
$$

which agrees with Lemmas 1 and 2.

\section{Conclusions}

A new direct method for order reduction has been proposed for PMDs describing a class of delay-differential control systems. The resulting PMD is associated with delay-differential systems of neutral type. The exact connection between the original PMD and the reduced firstorder PMD was set out and shown to be Fuhrmann's strict system equivalence. Furthermore, the reduced PMD can be easily computed from the original one using a trivial expansion followed by a finite sequence of elementary row and column operations. On the other hand, the transfer function and the zero structure of the original PMD are preserved making it possible to analyze the original system in terms of its reduced form. The resulting PMD may be larger in size than the one obtained by the method used by Pugh et al (1998a). However, the method presented in this paper has the advantage of providing a priori both the final reduced PMD and the transformation involved. 


\section{Acknowledgment}

The author wishes to express his thanks to Sultan Qaboos University (Oman) for their support in carrying out this research.

\section{References}

Boudellioua M.S. (2006): An equivalent matrix pencil for bivariate polynomial matrices. - Int. J. Appl. Math. Comput. Sci., Vol. 16, No. 2, pp. 175-181.

Byrnes C.I., Spong M.W. and Tarn T.J. (1984): A several complex variables approach to feedback stabilization of linear neutral delay-differential systems. — Math. Syst. Theory, Vol. 17, No. 2, pp. 97-133.

Fuhrmann P.A. (1977): On strict system equivalence and similarity. - Int. J. Contr., Vol. 25, No. 1, pp.5-10.

Johnson D.S. (1993): Coprimeness in multidimensional system theory and symbolic computation. - Ph.D. thesis, Loughborough University of Technology, UK.

Levy B.C. (1981): 2-D polynomial and rational matrices and their applications for the modelling of 2-D dynamical systems. - Ph.D. thesis, Stanford University, USA.

Pugh A.C., McInerney S.J., Boudellioua M.S. and Hayton G.E. (1998a): Matrix pencil equivalents of a general 2-D polynomial matrix. — Int. J. Contr., Vol. 71, No. 6, pp. $1027-$ 1050 .
Pugh A.C., McInerney S.J., Boudellioua M.S., Johnson D.S. and Hayton G.E. (1998b): A transformation for 2-D linear systems and a generalization of a theorem of Rosenbrock. Int. J. Contr., Vol. 71, No. 3, pp. 491-503.

Pugh A.C., McInerney S.J. and El-Nabrawy E.M.O. (2005a): Equivalence and reduction of 2-D systems. - IEEE Trans. Circ. Syst., Vol. 52, No. 5, pp. 371-275.

Pugh A.C., McInerney S.J. and El-Nabrawy E.M.O. (2005b): Zero structures of $n-D$ systems. — Int. J. Contr., Vol. 78, No. 4, pp. 277-285.

Pugh A.C., McInerney S.J., Hou M. and Hayton G.E. (1996): A transformation for 2-D systems and its invariants. Proc. 35th IEEE Conf. Decision and Control, Kobe, Japan, pp. 2157-2158.

Rosenbrock H.H. (1970): State Space and Multivariable Theory. — London: Nelson-Wiley.

Sebek M. (1988): One more counterexample in n-D systems Unimodular versus elementary operations. - IEEE Trans. Autom. Contr., Vol. AC-33(5), pp. 502-503.

Zerz E. (2000): Topics in Multidimensional Linear Systems Theory. — London: Springer.

Received: 1 November 2006 Revised: 18 February 2007 